\title{
Large Fibrovascular Polyp of the Hypopharynx With Dysphonia: Direct Laryngoscopic Removal
}

\author{
Abdulkadir Bucak ${ }^{\mathrm{a}, \mathrm{b}}$, Sahin Ulu , Abdullah Aycicek ${ }^{\mathrm{a}}$, Erdogan Okur ${ }^{\mathrm{a}}$, \\ Selcuk Sevinc ${ }^{\text {a }}$, Huseyin Isiklia, Abidin Duran ${ }^{a}$
}

\begin{abstract}
A 56-year-old male patient was admitted to our clinic with complaining of difficulty in swallowing and a mass voluntarily removed from the mouth. In his examination, a pedunculated mass which can be easily voluntary regurgitated, extending to the left hypopharyngeal region, completely disappearing with swallow, approximately $2 \mathrm{~cm}$ in size $7 \mathrm{~cm}$ in long was detected and totally excised by direct laryngoscopy. In this case report, large hypopharyngeal fibrovascular polyp was removed by direct laryngoscopy of a patient which was complained by dysphonia was presented.
\end{abstract}

Keywords: Fibrovascular polyp; Hypopharynx; Dysphonia

\section{Introduction}

Hypopharyngeal and esophageal fibrovascular polyps (FVP), which originate from the upper part of the gastrointestinal tract are rarely encountered benign tumors. Polyps are usually asymptomatic until they reach the size of recognized clinically [1]. The most common symptoms are difficulty swallowing and the feeling of foreign body. Specific feature of FVP's is regurgitation of a fleshy mass into mouth. Other complaints are difficulty in breathing, dysphonia and weight loss [2]. Surgical excision is the only treatment method [3].

In this report, the patient was admitted to our clinic with dysphonia and difficulty in swallowing depending on the mass which was grown over the time in the throat was presented.

\footnotetext{
Manuscript accepted for publication January 10, 2014

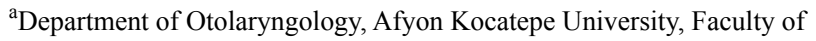
Medicine, 03200 Afyonkarahisar, Turkey

${ }^{\mathrm{b}}$ Corresponding author: Abdulkadir Bucak, Department of Otolaryngology, Afyon Kocatepe University, Faculty of Medicine, 03200 Afyonkarahisar, Turkey. Email: abdulkadirbucak@yahoo.com
}

doi: http://dx.doi.org/10.14740/jmc1037w

\section{Case Report}

A 56-year-old male patient was admitted to our clinic with the complaining of difficulty in swallowing and dysphonia, which was became more prominent in the last 6 months. He had a mass, which could easily regurgitated into the mouth. In his examination, a pedunculated mass which can be easily voluntary regurgitated, extending to the left hypopharyngeal region, completely disappearing with swallow, restricting the movement of his left vocal cord and arytenoid and approximately $2 \mathrm{~cm}$ in size $7 \mathrm{~cm}$ in long was detected (Fig. 1, 2 ). Video laryngoscopy of the patient was completely normal after swallowing the mass except reduced left vocal cord and arytenoid movements.

The mass, which reach to vallecula was detected at the localization of the left base of the tongue on preoperative computed tomography (CT) and magnetic resonance imaging (MRI) examination. The tough viscous mass, which approximately $7 \times 2 \times 1 \mathrm{~cm}$ in size and arised from the left piriform sinus-esophageal junction was totally excised by direct laryngoscopy.

Patient's complaints completely disappeared in the early postoperative period and recurrence was not detected for two-year follow-up period.

A fibroid polypoid tissue which covered up with non-

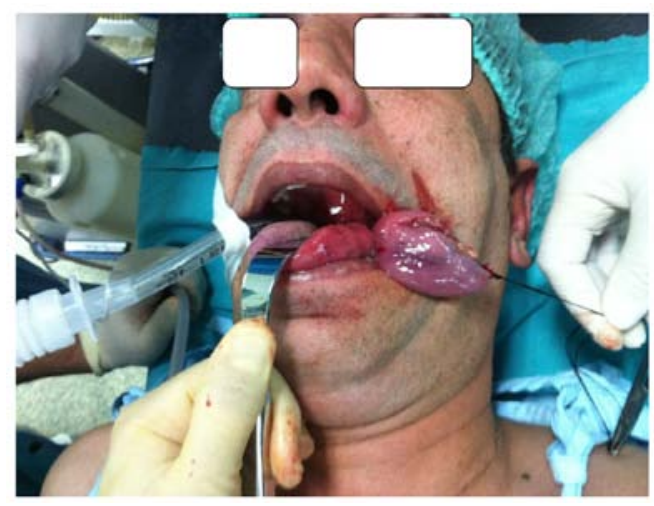

Figure 1. Pulling out the large pedunculated polyp measuring $7 \times 2 \times 1 \mathrm{~cm}$. 


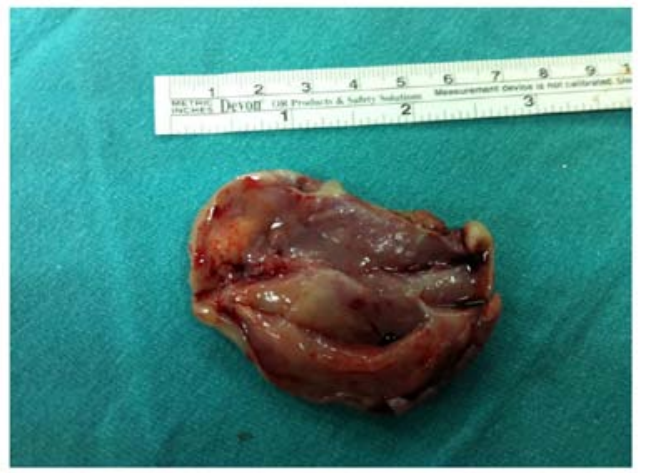

Figure 2. Gross appearance of the longitudinally incised fibrovascular polyp. The polyp core is composed of lobules of adipose tissue and bands of connective tissue.

keratinized stratified epithelium, blood vessels in the stroma and polymorphonuclear leukocyte, mononuclear inflammatory cell infiltration were found in the histopathological examination. There were no evidences of dysplasia or malignancy. The histopathological diagnosis was consistent with fibrovascular polyps (Fig. 3, 4).

\section{Discussion}

FVP of esophagus and hypopharynx are rarely encountered benign tumors of the upper digestive tract which are situated intraluminal and in the submucosal region. FVP of esophagus are 1 to $2 \%$ of all esophageal tumors and mostly localized in the proximal esophagus. Mostly was seen in men between the ages from 60 to 70 . Also, 5 month old infant with FVP has been reported $[4,5]$. FVP are often caused by two specific regions in posterior hypopharyngeal wall. One of them is called Killian space that is the region between the superior and inferior cricopharyngeal muscles and the other is so-called Laimer-Haeckermann space, that's the region between inferior cricopharyngeal muscle and proximal end

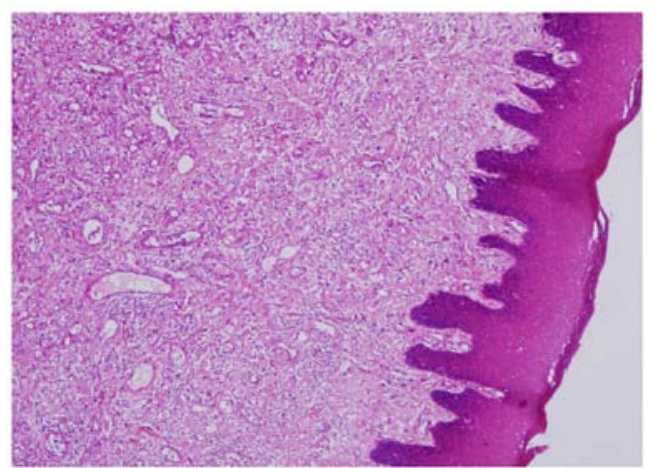

Figure 3. Polypoid tissue covered with non-keratinized stratified squamous epithelium (hematoxylin-eosin, original magnification $\times 40$ ).

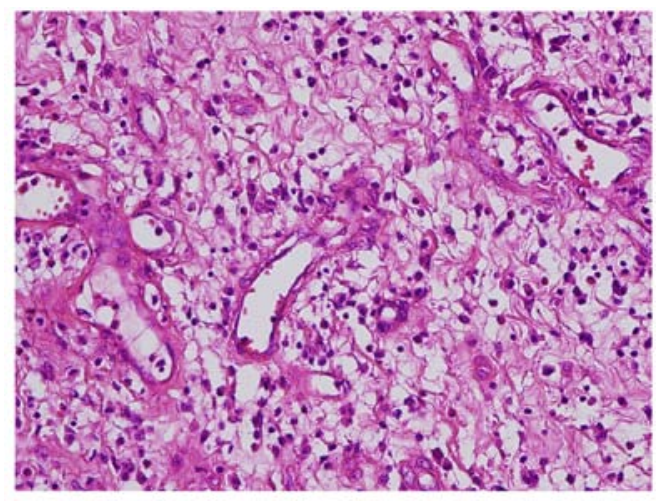

Figure 4. Inflammatory cell infiltration in fibrovascular stroma (hematoxylin-eosin, original magnification $\times 100$ )

of esophagus. These polyps are rarely originated from hypopharynx [1]. In our case, it was originated from the hypopharynx. Even if the underlying pathophysiology is not fully well understood, polyps begin as submucosal thickening and grow until they reach very large sizes by the influence of the traction force by swallowing and peristaltism [6]. FVP usually occur as solitary masses welded from the hypopharynx as well as multiple polyps have been reported in the literature [4].

The symptoms of FVP change according to the size, location and composed complications. These masses may remain asymptomatic for a long time until they reach to the symptomatic form [1]. The most common symptoms are progressive dysphagia and feeling of mass $[2,7]$. As in our case, voice change is rarely seen [8]. We think that this situation is due to the compression of the left hypopharyngeal polyp. Otherwise nonspecific respiratory symptoms such as chronic cough, dyspnea and recurrent pneumonia and emergency situations like as airway obstruction may be seen [1, $2,6]$. Regurgitation of the polyp to mouth is an engaging condition that can be approximately seen in half of the cases [9]. The mass is difficulty recognized when there is not regurgitation [2]. Occasionally, anemia due to occult gastrointestinal bleeding or hemorrhage when polyp folded onto itself can be seen [7]. CT, MRI, endoscopic ultrasonography, barium esophagogram and esophagogastroscopy can be used for diagnosis. Endoscopic diagnosis of hypopharyngeal FVP is easier from those who localized in esophagus. Nowadays, $\mathrm{CT}$ and MRI are the gold standards to demonstrate the content and the localization of the mass. Differential diagnosis of neoplastic or non-neoplastic pathologies such as hamartoma, inflammatory polyp, lipoma, hemangioma, lymphangioma, schwannoma, carcinoid tumor and kemodektoma can be done via histopathologic examination [10].

The $75 \%$ of the cases of PVP have been reported larger than $7 \mathrm{~cm}$ in the literature [6]. In our case, the mass was consistent with the literature as $7 \mathrm{~cm}$ in length.

The treatment of FVP is the surgical excision due to the 
progressive growth potential of the lesion and the risk of sudden death by reason of asphyxia. Local excision is sufficient for the treatment and recurrence is very rare [2, 4]. The most frequently preferred method is transcervical approach due to the originated anatomic region as well as transoral, transcervical, transthoracic resection can be preferred according to the tumor location and size [6]. The combination of transabominal and transcervical approach for resection due to size of the tumor has been presented in literature [4]. Nowadays, endoscopic surgery or direct laryngoscopy is recommended for polyps up to $2 \mathrm{~cm}$ as well as open surgery is recommended for larger than $8 \mathrm{~cm}$. In addition, it was suggested that these polyps can be removed via endoscopic approach $[1,2,4,6]$.

As a result, FVP of the hypopharynx may reach to very large sizes and regurgitate into the mouth. It may be the cause of voice changes and difficulty in swallowing. The FVP of the hypopharynx should be considered in the differential diagnosis.

\section{References}

1. Ozdemir S, Gorgulu O, Selcuk T, Akbas Y, Sayar C, Sayar H. Giant fibrovascular polyp of the hypopharynx: per-oral endoscopic removal. J Laryngol Otol. 2011;125(10):1087-1090.

2. Wang J, Han DM, Ni X, Ma LJ, Ye JY, Xiao Y. Fibro- vascular polyp of the hypopharynx and esophagus. Chin Med J (Engl). 2011;124(19):3182-3184.

3. Nishimura G, Horiuchi C, Yoshida T, Kawakami M, Yabuki K, Matsuda H, Mikami Y, et al. Fibromatous polyp of the hypopharynx. Auris Nasus Larynx. 2006;33(3):333-336.

4. I H, Kim JS, Shim YM. Giant fibrovascular polyp of the hypopharynx: surgical treatment with the biappoach. J Korean Med Sci. 2006;21(4):749-751.

5. Paik HC, Han JW, Jung EK, Bae KM, Lee YH. Fibrovascular polyp of the esophagus in infant. Yonsei Med J. 2001;42(2):264-266.

6. Pham AM, Rees CJ, Belafsky PC. Endoscopic removal of a giant fibrovascular polyp of the esophagus. Ann Otol Rhinol Laryngol. 2008;117(8):587-590.

7. Blacha MM, Sloots CE, Van Munster IP, Wobbes T. Dysphagia caused by a fibrovascular polyp: a case report. Cases J. 2008;1(1):334.

8. Ubukata H, Nakachi T, Kasuga N, Nagata H, Konishi S, Goto Y, Watanabe Y, et al. Esophageal fibrovascular polyp removed by cervical esophagotomy. Acta Gastroenterol Belg. 2010;73(4):514-516.

9. Paczona R, Ivan L, Jori J, Ivanyi B. Peroral endoscopic removal of a regurgitated giant polisegmented fibrovascular polyp of the esophagus. Diagn Ther Endosc. 2001;7(3-4):197-201.

10. Gupta M, Chaudhary N. Fibrovascular polyp of the oropharynx. Singapore Med J. 2011;52(2):e35-36. 